\title{
Expression and functional analysis of the propamocarb-related gene CSMAPEG in cucumber
}

\author{
Fan Zhang, Zhiwei Qin ${ }^{*} \mathbb{D}$, Xiuyan Zhou, Ming Xin, Shengnan Li and Jie Luan
}

\begin{abstract}
Background: Propamocarb (PM) is one of the main pesticides used for controlling cucumber downy mildew. However, due to its volatility and internal absorption, PM can easily form pesticide residues on cucumber fruits that seriously endanger human health and pollute the environment. The breeding of new cucumber varieties with a low abundance of PM residues via genetic methods constitutes an effective strategy for reducing pesticide residues and improving cucumber safety and quality. To help elucidate the molecular mechanism resulting in a low PM residue abundance in cucumber, we used the cucumber cultivar 'D0351' (which has the lowest PM residue content) as the test material and identified genes related to low PM residue abundance through high-throughput tag-sequencing (Tag-Seq).

Results: CSMAPEG was constitutively expressed and showed both varietal and organizational differences. This gene was strongly expressed in 'D0351'. The expression levels of CSMAPEG in different cucumber tissues under PM stress were as follows: fruit>leaf>stem>root. CSMAPEG can respond to salicylic acid (SA), gibberellin (GA) and Corynespora cassiicola Wei (Cor) stress and thus plays an important regulatory role in plant responses to abiotic and biological stresses. The PM residue abundance in the fruits of CSMAPEG-overexpressing plants was lower than those found in antisense CSMAPEG plants and wild-type plants at all tested time points. The results revealed that CSMAPEG played a positive role in reducing the PM residue abundance. A CSMAPEG sense construct increased the contents of SOD, POD and GST in cucumber fruits, enhanced the degradation and metabolism of PM in cucumber, and thus effectively reduced the pesticide residue abundance in cucumber fruits.

Conclusions: The expression patterns of CSMAPEG in cucumber cultivars with high and low pesticide residue abundances and a transgenic verification analysis showed that CSMAPEG can actively respond to PM stress and effectively reduce the PM residue abundance in cucumber fruits. The results of this study will help researchers further elucidate the mechanism responsible for a low PM residue abundance in cucumber and lay a foundation

for the breeding of new agricultural cucumber varieties with low pesticide residue abundances.
\end{abstract}

Keywords: Cucumber, Cucumber downy mildew, Propamocarb, Functional analysis, CSMAPEG

\section{Background}

Cucumber is a staple vegetable in China that is cultivated throughout the country, primarily through protected cultivation. Because of the special microclimate in the protected cultivation environment, continuous cropping and other factors, downy mildew is commonly observed during cucumber production. Cucumber downy mildew can

\footnotetext{
* Correspondence: qzw303@126.com

College of Horticulture and Landscape Architecture, Key Laboratory of Biology and Genetic Improvement of Horticultural Crops (Northeast Region), Northeast Agricultural University, Harbin 150030, China
}

be found in plants from the seedling to adult stages, and severe cases can cause yield losses of 20-40\% [1]. Therefore, high levels of propamocarb (PM) are used to control this disease. Due to the lack of suitable substitutes for controlling cucumber downy mildew, nearly $80 \%$ of farmers in China use PM to control cucumber downy mildew [2]. $\mathrm{PM}$ is a low-toxicity fungicide that can effectively control cucumber downy mildew. However, due to its volatility and internal absorption, PM can pollute the environment and easily form pesticide residues on cucumber fruit [3], and these pesticide residues on cucumber are regarded as 
neurotoxicants that seriously endanger human health. Animal experiments have confirmed that pesticide residues exert carcinogenic effects [4-7], and a pathogenic analysis has revealed that the fungicide PM (carbamate) can inhibit the decomposition of acetylcholinesterase to induce the accumulation of acetylcholine in the body, which would affect the normal nerve conduction process in organisms and even lead to the poisoning and death of organisms [8]. Therefore, PM residues on crops have attracted increasing attention due to food safety and health concerns. A system for the evaluation of pesticide residues in cucumber germplasm resources has been established, and the botanical morphological characteristics have been clarified. Germplasm resources with low and high residue abundances of the pesticides deltamethrin, PM and myclobutanil have been identified [9]. Previous studies have performed genetic analyses of cucumber PM residue, have revealed that the abundance of PM residues in cucumber fruits is a quantitative trait controlled by multiple genes, and have detected a QTL related to PM residue abundance [10]. The fluidity and changes in the PM residue abundance in plants have also been analyzed. Varieties with a low pesticide residue abundance exhibit a lower residue quantity and a decreased rate of change in the residue quantity compared with varieties with a high pesticide residue abundance [11]. Based on the results from physiological and biochemical studies of cucumbers with low PM residue abundances, the differentially expressed genes in cucumber fruits under PM stress have been screened through Solexa and comparative genomics methods. The identified genes can be divided into four main categories: (1) transcription factors involved in transcriptional regulation, such as WRKY and DREB; (2) pesticide detoxification-related genes, such as the $A B C$ family, cytochrome P450 family, and GSTs; (3) signal transductionrelated genes, such as aminotransferase and succinate dehydrogenase; and (4) stress- and stimulation-related genes, such as heat shock proteins (HSPs) and cysteine synthase (CYS) [12]. Studies of the CsABC19 [13], CsWRKY30 [14], CsSDH [15] and CsDIR16 [16] genes, which respond to PM stress in variants with high and low PM residue abundances, have shown that these differentially expressed genes can significantly improve the resistance of transgenic Arabidopsis thaliana or cucumber to PM stress.

Glutathione (GSH) is involved in biological transformations and promotes the transformation of harmful toxins in the body into harmless substances that are then excreted. GSH can directly participate in metabolic reactions as a substrate. This molecule also induces the expression of detoxification genes, activates detoxification enzymes, such as GST, in plants, promotes detoxification and metabolic reactions, and thus effectively reduces the abundance of pesticide residues in plants $[17,18]$. In vivo studies have revealed that GSH-related pathways play an important role in pesticide degradation and metabolism in maize, wheat and tomato plants [19-21]. Reactive oxygen species (ROS) can be produced by plants under stress, and their production can lead to the peroxidation of membrane lipids to malondialdehyde (MDA). After MDA enters cells, it can react with proteins and nucleic acids and thereby induce losses in the functions of these compounds. Therefore, the MDA content can reflect the degree of damage in plants under stress. However, GST can eliminate ROS to maintain normal life metabolism activities in plants [22-24]. Protective enzymes (mainly SOD and POD) are found in the membrane system of cucumber. SOD can catalyze the disproportionation of $\mathrm{O}_{2}$ to $\mathrm{H}_{2} \mathrm{O}_{2}$, and POD can reduce $\mathrm{H}_{2} \mathrm{O}_{2}$ to $\mathrm{H}_{2} \mathrm{O}$, can efficiently scavenge oxygen free radicals and thus increase the metabolic capacity of plants for toxic substances [25]. The metabolic membrane-related proteins that form part of MAPEG family include GSH and arachidonic acid. Most members of the MAPEG family, which are related to cell detoxification and drug resistance, are involved in the synthesis of the inflammatory mediators LTs and PGE2 and in the promotion of in vivo anti-electron affinity, oxygen free radical attack, and exogenous lipid metabolism. Studies have shown that MAPEG proteins regulate liver injury in mice and beagles and exert detoxification effects $[26,27]$. In recent years, toxicology and pharmacology research on the functions of the MAPEG family has rapidly progressed from the known involvement of this family in arachidonic acid and GSH metabolism, but the detoxification mechanism of the MAPEG family proteins in plants remains unclear.

Thus far, many genes related to drug detoxification have been identified. To study the role of these detoxification-related genes in the detoxification of fungicides in cucumber, Solexa high-throughput tag sequencing was performed [12], and the results were used to identify the candidate gene Cucsa.034680.1 involved in the GSH pathway. This gene, which is named CsMAPEG, belongs to the MAPEG protein family. The results reported by $\mathrm{Wu}$ Peng showed that the activity of glutathione transferase is significantly increased after treatment with PM [2]. A fluorescence quantitative analysis previously showed that CSMAPEG is upregulated in the low-pesticide-residue-abundance $\mathrm{cu}$ cumber cultivar 'D0351' under PM stress and could exhibit a significant response to PM threat. However, the mechanism underlying the response of CSMAPEG to PM stress has not been reported. The cloning of CsMAPEG using 'D0351' fruit cDNA as the template and a bioinformatics analysis of the sequence domains and gene structure were performed to explore the physicochemical properties of this protein as well as its homologous sequences and subcellular localization. The expression of CsMAPEG in strains with high and low abundances of PM, the residual quantity of PM in CsMAPEG-overexpressing plants and 
the related physiological and biochemical indexes were analyzed. The aims of this study were to explore the performance of CSMAPEG under PM stress and to help elucidate the mechanism underlying the production of low abundances of PM residues in cucumber, and the results will contribute to the breeding of new cucumber varieties with a low abundance of pesticide residues.

\section{Results}

\section{Cloning and bioinformatics analysis of CSMAPEG}

CsMAPEG was amplified by PCR using CSMAPEG-F and CSMAPEG-R as the primers and cucumber 'D0351' fruit cDNA as the template (Fig. 1a). The CSMAPEG sequence was then confirmed by PCR and repeated sequencing (Fig. 1b). Full-length CsMAPEG is $438 \mathrm{bp}$ in length and encodes 145 amino acids (Fig. 1c). Our sequence was consistent with the coding region of the gene (Csa5G409710.1) obtained from the alignment of sequences in the cucumber genome database (http://www.icugi.org/).

The amino acid sequence alignment of CSMAPEG showed that the protein has one MAPEG domain (Additional file 3: Figure S1) and belongs to the MAPEG superfamily; thus, the protein was named CsMAPEG. The analysis of CsMAPEG revealed a predicted theoretical molecular weight of $16.486 \mathrm{kDa}$, a theoretical isoelectric point of 9.66 , an atomic composition of $\mathrm{C}_{775} \mathrm{H}_{1193} \mathrm{~N}_{189} \mathrm{O}_{186} \mathrm{~S}_{11}$, a total number of atoms of 2354, and a fat coefficient of 104.83. The highest hydrophobicity scores of the protein encoded by this gene (Additional file 4: Figure S2) were found to equal 2.944 at the 16th amino acid position and 2.122 at the 67th amino acid position, and the average hydrophilic coefficient was estimated as -0.538 , which indicated that the protein is hydrophilic. A clear signal peptide cleavage site was not detected for this protein (Additional file 5: Figure S3), which suggested that CsMAPEG might be a cytoplasmic matrix or organelle matrix protein but not a membrane or secretory protein. The prediction of the transmembrane domain of the CsMAPEG protein showed three distinct transmembrane regions (Additional file 6: Figure S4). The secondary structure of the protein (Additional file 7: Figure S5) was analyzed, and the analysis revealed 83 alpha helices, which accounted for $57.24 \%$ of the total polypeptide chain, 20 extended main chains, which accounted for $13.79 \%$ of the whole polypeptide chain, and 36 random coils, which accounted for $24.83 \%$ of the whole polypeptide chain. The protein sequence was submitted to the protein homology modeling program Phyre to predict the three-level structure of the protein (Additional file 8: Figure S6).

The cucumber genome database was searched for Csa5G409710.1 to obtain the complete gene sequence, and a region $2000 \mathrm{bp}$ upstream was selected as the promoter sequence. The analysis results are shown in Additional file 1: Table S1. In addition to the TATA and CAAT elements inherent in the eukaryotic promoter and some common light-responsive elements, such as the 3-AF1-binding site, GT1 motif and SP1binding site, the promoter also contains an ARE anaerobic induction element, a P-box gibberellin (GA) response element, an ERE ethylene response element, a TC-rich repeat defense and stress response element, three heat shock reaction elements, one TCA-element salicylic acid (SA) response element, one AuxRR-core auxin response element and other cis-elements.

\section{Phylogenetic tree of CSMAPEG}

The amino acid sequences of MAPEG family proteins from 10 different specieswere downloaded from the NCBI database (https://www.ncbi.nlm.nih.gov/) for homology alignment. A phylogenetic tree was constructed using the neighbor-joining (NJ) method with MEGA software (version 5.2). The results are shown in Additional file 9: Figure S7, and the scale bar indicates the average number of amino acid substitutions per site. Fifteen family members (Additional file 2: Table S2), including CsMAPEG, were grouped into

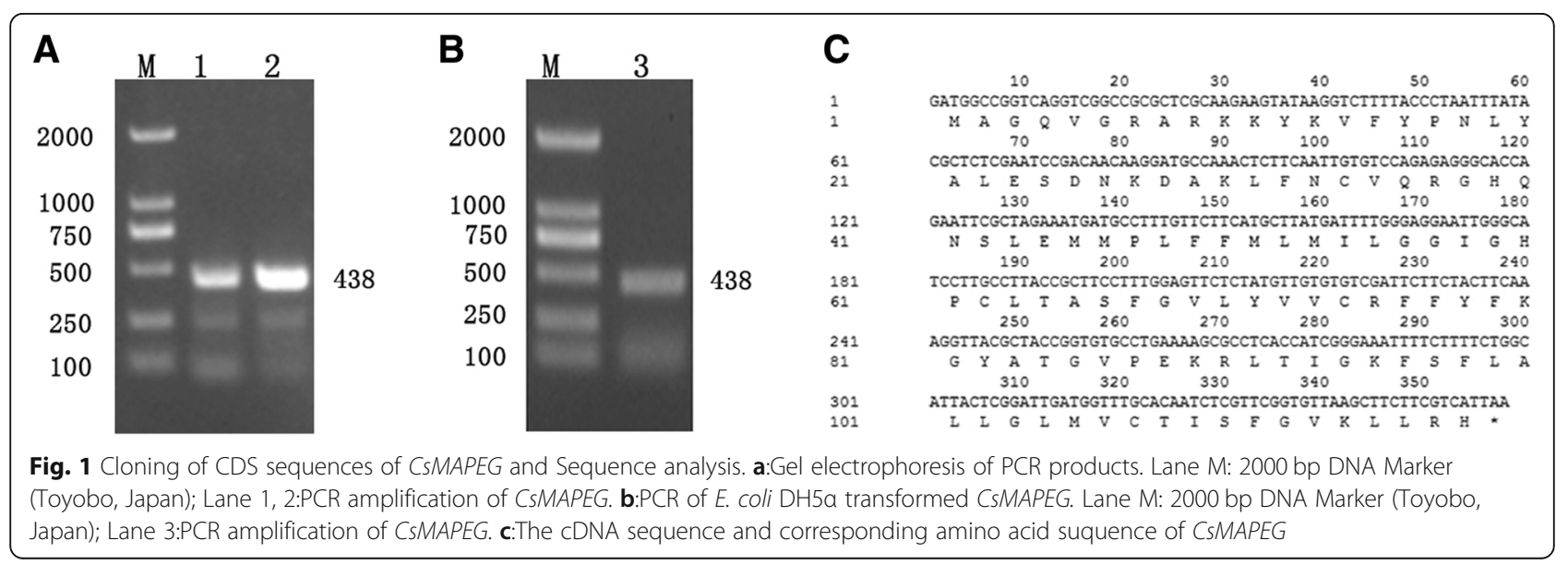


two groups. The MAPEG protein shows strong conservation among different species, and the proteins from plants in the same family and genus show high similarity. CsMAPEG and the protein from melon Cucumis melo L. (XM008462283.2) form a small evolutionary branch and show the closest relationship; in addition, these proteins exhibit a distance of less than 0.1 from the proteins of balsam pear Momordica charantia L. (XM022285675.1) and pumpkin Cucurbita moschata (XM023137096.1), which belong to Cucurbitaceae. The change in nucleotides among these proteins is small, and their homology is relatively high.

\section{Subcellular localization of the CSMAPEG protein}

The 35S:CsMAPEG-GFP fusion expression vector and the pGII-EGFP empty vector were introduced into Arabidopsis protoplasts. The subcellular localization of the CsMAPEG fusion protein was observed using a confocal laser microscope, and the results are shown in Fig. 2. Bright green fluorescence was observed in whole cells transfected with the empty vectors, whereas enriched green fluorescence following transfection with the CsMAPEG-pGIIEGFP fusion expression vector was observed only in the cytoplasm. Therefore, the results clearly indicated that CsMAPEG is localized in the cytoplasm.

\section{Expression pattern of CSMAPEG in response to PM treatment}

The expression patterns of CsMAPEG in cucumber plants treated with PM showed differences between the low-PMresidue-abundance cultivar 'D0351' and the high-PM-residue-abundance cultivar 'D9320' (Fig. 3). Exposure to PM increased the expression levels of CSMAPEG in the roots, stems, leaves and fruits of 'D0351' over time compared with the expression levels found in the same tissues of 'D9320'. At all the tested time points, significantly higher CsMAPEG expression was found in 'D0351' roots compared with the control group, and specifically, the expression level in the 'D0351' roots at $24 \mathrm{~h}$ was 2.69-fold higher than that the control level (Fig. 3a). However, CsMAPEG expression in 'D9320' was significantly downregulated at $48 \mathrm{~h}$, and the expression levels at the other time points were not significantly different from those of the control group (Fig. 3b). The expression levels of CSMAPEG in the stems of 'D0351' were first upregulated and then downregulated. The relative expression of CSMAPEG in the 'D0351' stems at each time point was significantly higher than that in the control group and reached its maximal level, which was 3.24-fold higher than that of the control group, at $24 \mathrm{~h}$ (Fig. 3c). The levels in 'D9320' were decreased significantly compared with those of the control at all the tested time points with the exception of 1 and 6 $\mathrm{h}$, and the overall expression level showed a smooth change and was significantly lower than the expression level in 'D0351' (Fig. 3d). The expression of CSMAPEG in the leaves of 'D0351' was upregulated at all tested time points compared with that of the control. The relative expression level reached its maximum at $48 \mathrm{~h}$, and this maximal level was 2.61-fold higher than the control level (Fig. 3e). However, CsMAPEG expression in 'D9320' leaves was downregulated at all time points; in addition, the change in CSMAPEG expression in 'D9320' leaves was stable, and the levels were significantly lower than those in 'D0351' (Fig. 3f). The patterns of CsMAPEG expression in 'D0351' and 'D9320' fruits were basically consistent, characterized by an initial increase and a subsequent decrease, although

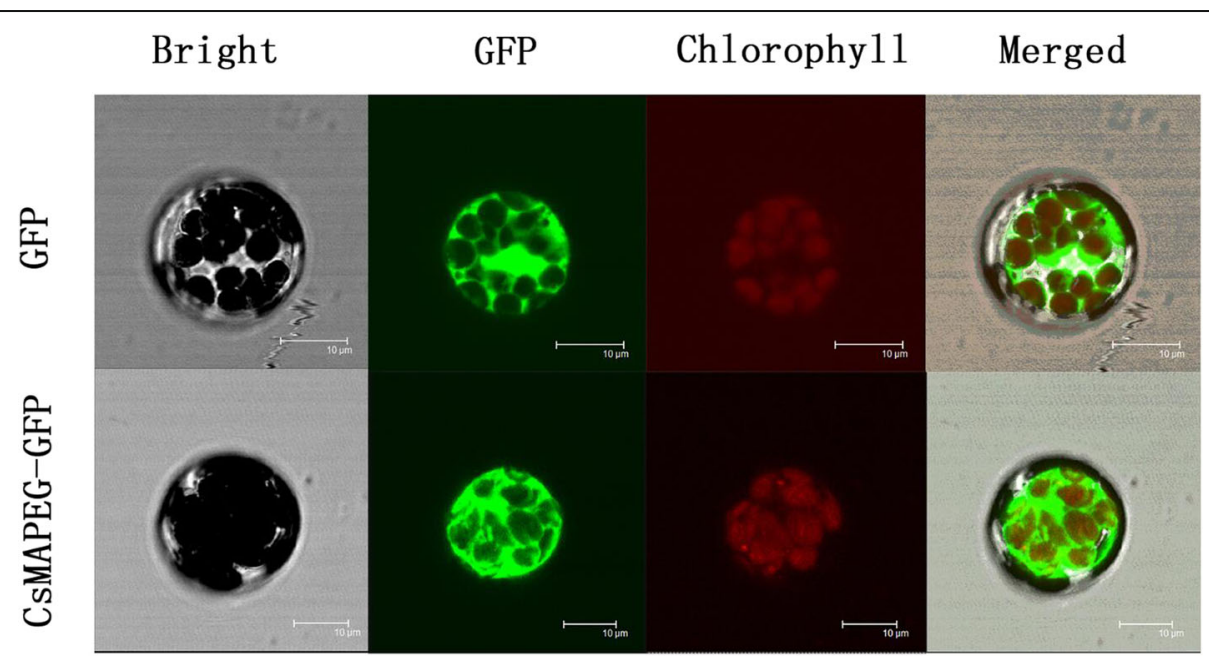

Fig. 2 Subcellular localization of CSMAPEG-pGII-EGFP protein in Arabidopsis protoplasts. Subcellular localization of the CSMAPEG-pGII-EGFP fusion protein in Arabidopsis protoplasts. Images show protoplasts prepared from 3- to4-week-old Arabidopsis leaves expressing CsMAPEG-pGII-EGFP (bottom row) or pGII-EGFP (upper row). Bright-field illumination, GFP fluorescence, chlorophyll fluorescence, and an overlay of GFP and chlorophyll fluorescence are shown. Scale bars, $10 \mu \mathrm{m}$ 

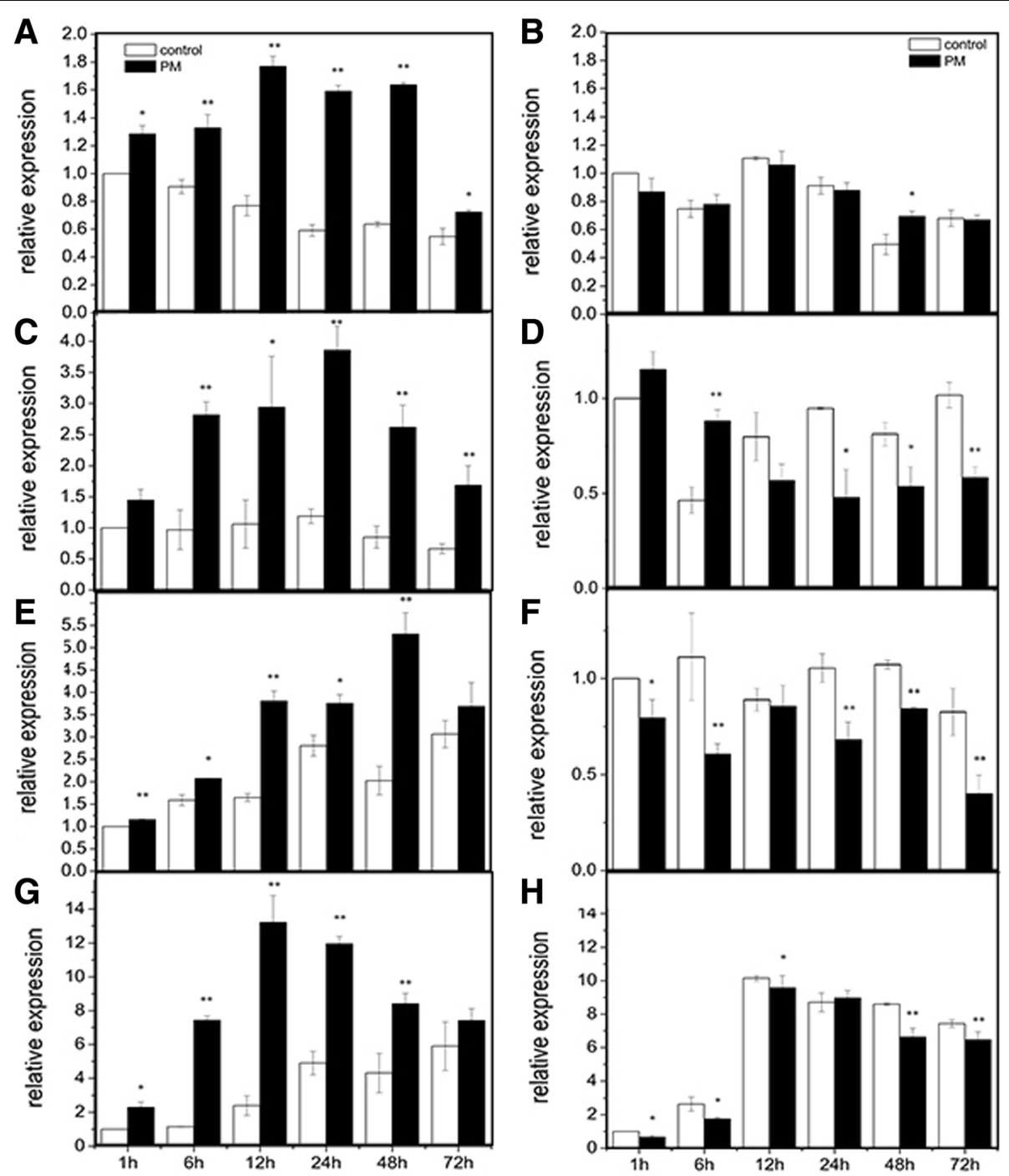

Fig. 3 Expression pattern of CSMAPEG in response to propamocarb stress. a: roots of D0351; b:roots of D9320; c:stem of D0351; d:stem of D9320; e:leaf of D0351; f:leaf of D9320; g:fruit of D0351; $\mathbf{h}$ :fruit of D9320; the "** presents the value is extremely significant at 0.05 level based on student t test; the "***" presents the value is extremely significant at 0.01 level based on student $t$ test

the gene expression level in 'D0351' at all tested time points was significantly higher than that in 'D9320'. The relative expression of CSMAPEG in 'D0351' fruits increased rapidly within $6 \mathrm{~h}$ and reached its maximum at $12 \mathrm{~h}$, and this maximal expression level was 5.49-fold higher than that of the control group. After $12 \mathrm{~h}$, the expression level decreased, although the values remained higher than those of the control at all time points (Fig. 3g). The CsMAPEG expression levels in 'D9320' fruits were upregulated only at 6 and $24 \mathrm{~h}$, and these values were 1.81and 1.34-fold higher than those of the control group, respectively. The relative expression of CsMAPEG at the other time points was lower than that of the control group (Fig. 3h). The CsMAPEG expression levels in different organs of the low-pesticide-residue-abundance cultivar 'D0351' were ordered as follows: fruit>leaf>stem>root. The expression of CsMAPEG in the stems and leaves of 'D0351' was significantly higher than that in the same parts of 'D9320'. CsMAPEG was specifically expressed in the stems and leaves of 'D0351' compared with those of 'D9320'.

\section{Analysis of CSMAPEG expression in response to different external factors}

The expression patterns of CsMAPEG after 'D0351' and 'D9320' seedlings at the three-true-leaf stage were treated with the hormones jasmonic acid (JA), SA and GA were analyzed. The results (Fig. 4a and b) showed that SA induction significantly upregulated the expression of CSMAPEG in the low-PM-residue-abundance cultivar 'D0351' to a level that was 8.76-fold higher than that of the control. The expression of CsMAPEG in the 

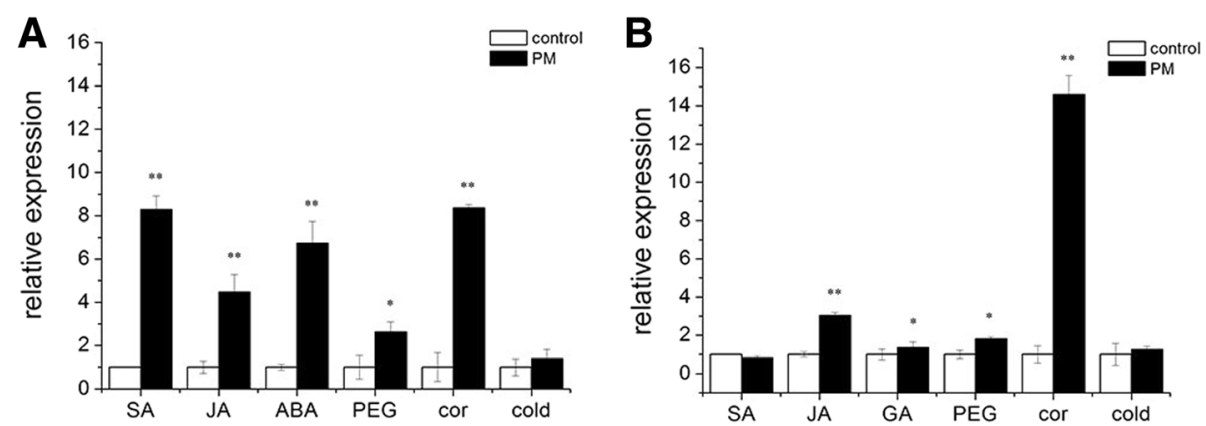

Fig. 4 Expression pattern of CSMAPEG under different stress of 'D0351' and 'D9320'. a:Cucumber variety 'D0351'; b:Cucumber variety 'D9320'.the "**" presents the value is extremely significant at 0.05 level based on student $t$ test; the "**" presents the value is extremely significant at 0.01 level based on student $t$ test

high-PM residue-abundance cultivar 'D9320' was not significantly different from that of the control. The JAinduced expression patterns of CSMAPEG in 'D0351' and 'D9320' were similar and showed very significant upregulation, although the relative expression of CsMA$P E G$ in 'D0351' was higher than that in 'D9320'. The expression of CsMAPEG in 'D0351'was 4.47-fold higher than that of the control and 3.03-fold higher than that of the 'D9320' cultivar. After GA treatment, CsMAPEG showed different expression patterns in 'D0351' and 'D9320'. Its expression was upregulated significantly in 'D0351' to a value that was 6.73-fold higher than that in the control, and this value was only slightly higher than that in 'D9320', which presented a relative expression level that was 1.36 -fold higher than that in the control. In 'D0351', CsMAPEG expression was upregulated after $\mathrm{SA}$, JA and GA induction. In contrast, in 'D9320', CsMAPEG expression was upregulated only after JA treatment and did not show significant changes after the SA and GA treatments. However, a significant difference in the CSMAPEG expression pattern was found between 'D0351' and 'D9320'. To study the function of the MAPEG family in stress tolerance, we subjected seedlings of 'D0351' and 'D9320' at the three-true-leaf stage to drought, Cor and low temperature stress and analyzed the resulting expression patterns of CSMAPEG. As shown in Fig. 4, CsMAPEG expression in 'D0351' and 'D9320' under PEG stress was upregulated, and these upregulated levels were significantly different from that of the control. The relative expression of CSMAPEG in 'D0351' was significantly higher than that in 'D9320', and the levels in these strains were 2.63- and 1.83-fold higher than that in the control, respectively. The expression of CSMAPEG was significantly upregulated in 'D0351' and 'D9320' under Cor stress. The expression of CSMAPEG in these strains was 14.60 - and 8.36-fold higher than that in the control, respectively. Under low temperature stress, the expression of CsMAPEG in 'D0351' and 'D9320' was nearly equivalent, and no significant difference compared with the control was detected.

\section{Construction of the CSMAPEG expression vector and genetic transformation of cucumber}

We generated the overexpression vectors CsMAPEG(+)PCXSN and CsMAPEG(-)-PCXSN under the control of the strong constitutive CaMV35S promoter (Fig. 5a). The overexpression vectors CSMAPEG(+)-PCXSN and CSMAPEG(-)-PCXSN were successfully transferred into 'D0351' and 'D9320' using cucumber genetic transformation technology (Additional file 10: Figure S8). The total DNA of the resistant plants was used as a template, the pCXSN-CsMAPEG(+) plasmid was used as a positive control, and water was used as a negative control. The primers used for PCR detection were specific for the pCXSN-1250 vector. As shown in Fig. 5b and c, the target fragments were approximately $500 \mathrm{bp}$ in the positive control and some resistant plants, although no specific bands were found in the negative control, which indicated that the pCXSN-CsMAPEG plasmid had been successfully integrated into the cucumber genome. To eliminate false positives in the resistant plants and ensure the integrity and accuracy of the experiment, we extracted RNA using the TRIzol method and identified the sequence by qRT-PCR. The normal expression of CSMAPEG in cucumber was affected by the transfection of exogenous genes, and the expression level was changed. The expression patterns were significantly different. The three CSMAPEG-overexpressing plants with the highest levels of CSMAPEG were selected for the detection of PM residues and the determination of physiological and biochemical indexes. In 'D0351', the expression of CSMAPEG was significantly upregulated after the transfer of CsMAPEG(+), and the CsMAPEG expression level in the T0 and T1 plants was 11.12- and 8.72-fold higher than that in the wild-type, respectively. After the transfer of CsMAPEG(-), the expression of CsMAPEG in the T0 and T1 plants was downregulated 


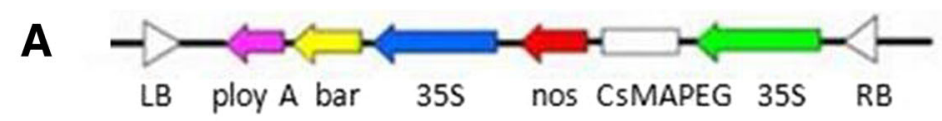

B

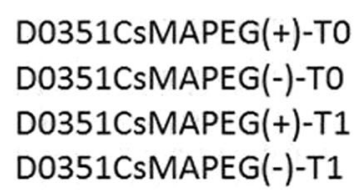

D

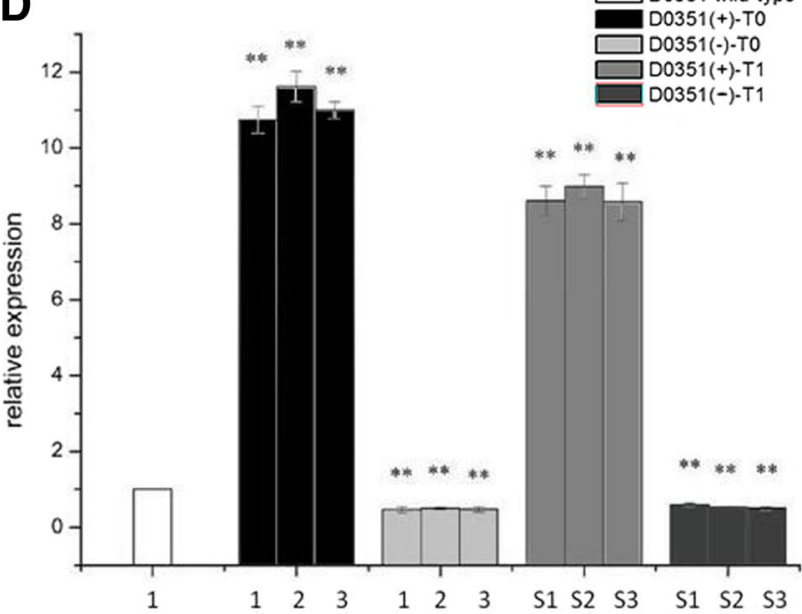

C
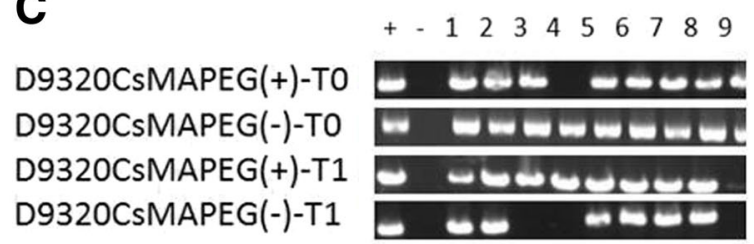

Fig. 5 Molecular biological verification of transgenic plants. a: Construction of plant vector pCXSN-CSMAPEG;b: 'D0351'transgenic plants $T_{0}$ and $T_{1}$ were identified by PCR; c: 'D9320'transgenic plants $T_{0}$ and $T_{1}$ were identified by PCR; d: Relative transcript levels of CsMAPEG in 'D0351'transgenic plants $\mathrm{T}_{0}$ and $\mathrm{T}_{1} ; \mathbf{e}$ : Relative transcript levels of CsMAPEG in 'D9320' transgenic plants $\mathrm{T}_{0}$ and $\mathrm{T}_{1}$

to levels that were 0.48 - and 0.55 -fold of the wild-type level (Fig. 5d). In 'D9320', the expression of CsMAPEG was upregulated after the transfer of CsMAPEG(+), and the expression levels in the T0 and T1 plants were approximately 6.98 - and 5.41 -fold higher than that of the wild-type. After the transfer of CsMAPEG(-), the expression levels of CSMAPEG in the T0 and T1 plants were approximately 0.48 - and 0.79 -fold of the wild-type level (Fig. 5e).

\section{Analysis of PM residues in CSMAPEG-overexpressing plants}

'D9320' plants with similar expression levels of CsMAPEG were identified by PCR and qRT-PCR, and the PM residues in fruits after treatment with $\mathrm{PM}$ were determined (Fig. 6). The levels of residues in CsMAPEG(+)overexpressing cucumber fruits at six time points from 1 to $72 \mathrm{~h}$ were significantly lower in the 'D9320' T0generation fruits compared with the wild-type control fruits, and the effect was extremely significant at 12 and $72 \mathrm{~h}$, with levels that were 0.67 - and 0.80 -fold of those found in the wild-type fruits, respectively. The average from the six time points obtained from the 'D9320' T0generation fruits was $0.81 \mathrm{mg} / \mathrm{kg}$, which was $0.26 \mathrm{mg} / \mathrm{kg}$ lower than the average value found for the wild-type fruits. The results showed that the CsMAPEG(+) transfer could effectively reduce the PM residues in fruits. The residue abundance in the CsMAPEG(-) transgenic plants at four time points from $12 \mathrm{~h}$ to $72 \mathrm{~h}$ was significantly or extremely significantly higher than that in the wild-type plants. The highest residue level of $1.86 \mathrm{mg} / \mathrm{kg}$ was found at $24 \mathrm{~h}$. The residue abundances in the CSMA$P E G(-)$ plants at 24 and $72 \mathrm{~h}$ were extremely significantly higher than those of the wild-type, and these values were 1.76- and 1.48-fold higher than those in the wild-type, respectively. The average residue abundance in the CsMAPEG(-) transgenic cucumber fruits at six time points was $1.37 \mathrm{mg} / \mathrm{kg}$, which was $0.29 \mathrm{mg} / \mathrm{kg}$ higher than that found for the wild-type plants.

The PM residue abundance in the CSMAPEG(+)-overexpressing, CsMAPEG(-)-overexpressing and wild-type plants at the T1 generation showed the same trend; specifically, 

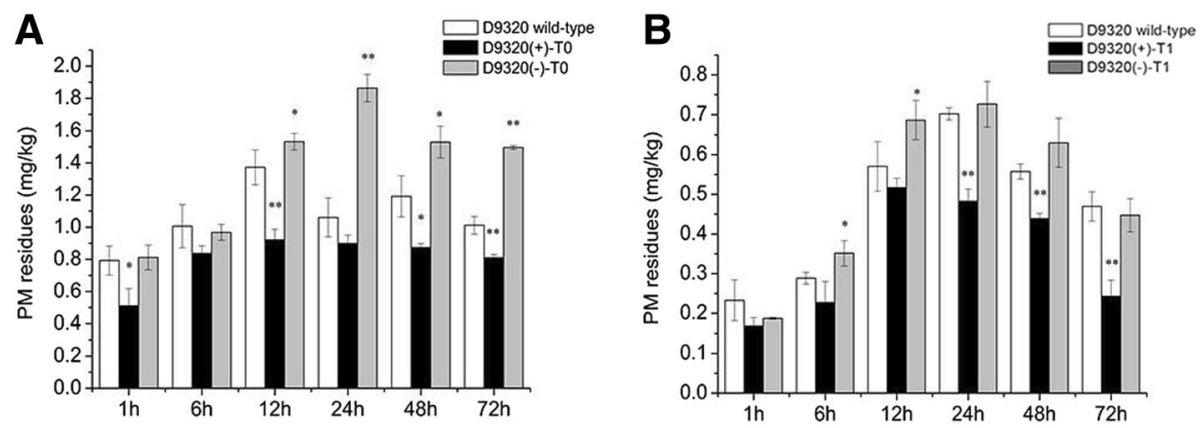

Fig. 6 CSMAPEG residue of 'D9320' transgenic plant. a: Quantitative analysis of PM residues in 'D9320' $T_{0}$ transgenic plants using gas chromatography; $\mathbf{b}$ : Quantitative analysis of PM residues in 'D9320' $T_{1}$ transgenic plants using gas chromatography. The "*" presents the value is extremely significant at 0.05 level based on student $t$ test; the "**" presents the value is extremely significant at 0.01 level based on student $t$ test

the PM residue abundance first increased and then decreased over time. Compared with the T0 plants, these T1 plants showed decreased PM residue abundances at all tested time points. The PM residue abundances in the CSMAPEG(+)-overexpressing plants were lower than those in the wild-type plants at the six tested time points. Specifically, the residue levels in the CSMAPEG(+)-overexpressing plants were 0.69-, 0.79- and 0.52-fold of those of the control plants at 24, 48 and $72 \mathrm{~h}$, respectively, and these differences were significant. The average value from six time points was $0.35 \mathrm{mg} / \mathrm{kg}$, which was $0.12 \mathrm{mg} / \mathrm{kg}$ lower than the average value obtained for the wild-type. The PM residue abundance in the CSMAPEG(-)-overexpressing T1 plants first increased significantly, reached a maximum of $0.73 \mathrm{mg} / \mathrm{kg}$ at $24 \mathrm{~h}$, and then decreased slowly. The PM residues in these plants were higher than those in the wild-type at all time points with the exception of 1 and $72 \mathrm{~h}$, when slightly lower residue abundances were detected in these plants relative to the wild-type plants. The average residue abundance of the CsMAPEG(-) transgenic cucumber fruit was $0.50 \mathrm{mg} / \mathrm{kg}$, which was $0.03 \mathrm{mg} / \mathrm{kg}$ higher than that in the wild-type plants.

'D0351' plants with similar expression levels of CsMAPEG were identified by PCR and qRT-PCR, and the PM residues in fruits after treatment with PM were determined
(Fig. 7). The residue abundances in CsMAPEG(+)-overexpressing cucumber fruits at six time points from 1 to $72 \mathrm{~h}$ were lower in the 'D0351' T0-generation fruits than in the wild-type fruits. The maximum residue abundance of 0.09 $\mathrm{mg} / \mathrm{kg}$ was observed at $12 \mathrm{~h}$. The residue abundances at 6 , 24 and $72 \mathrm{~h}$ were $0.85-, 0.80$ - and 0.78 -fold of those of the wild-type plants, respectively, and these differences were significant. In addition, the residue abundances in the CSMAPEG(+) plants at 1 and $12 \mathrm{~h}$ were extremely significantly lower than those in the wild-type plants, and the values obtained for the CSMAPEG(+) plants were 0.71- and 0.67 -fold of the wild-type values, respectively. The average value from six time points was $0.07 \mathrm{mg} / \mathrm{kg}$, which was 0.02 $\mathrm{mg} / \mathrm{kg}$ lower than the average value obtained for the wildtype. The results showed that $\operatorname{CSMAPEG(+)}$ transfer could significantly reduce the PM residue abundance in cucumber fruits. The residue abundances in the CsMAPEG(-)overexpressing cucumber fruits were significantly higher than those in the wild-type plants at six time points from 1 to $72 \mathrm{~h}$, with the exception of 1 and $12 \mathrm{~h}$, when slightly lower values were detected. The average residue abundance in the CsMAPEG(-)-overexpressing plants was $0.09 \mathrm{mg} / \mathrm{kg}$, which was $0.01 \mathrm{mg} / \mathrm{kg}$ higher than that obtained for the wild-type. The residue abundances in these plants at 6 and $72 \mathrm{~h}$ were 1.11- and 1.28-fold higher compared with the
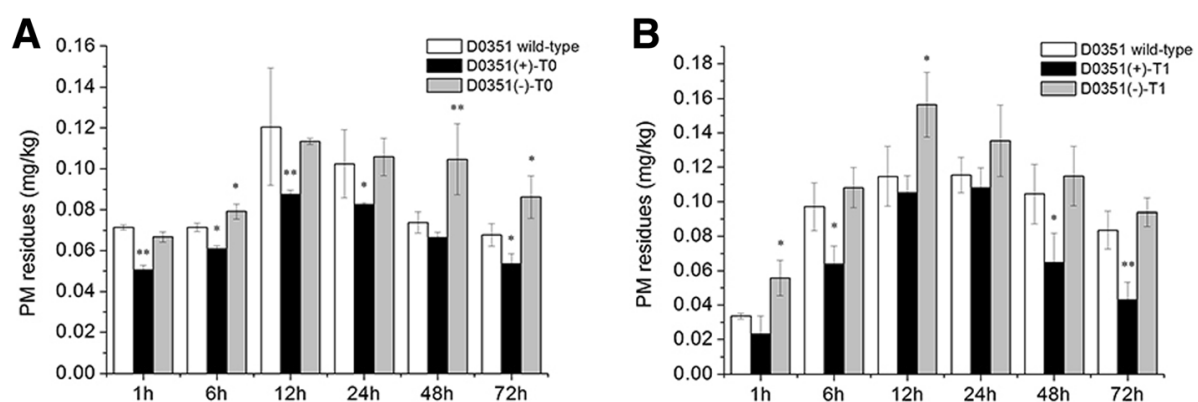

Fig. 7 CSMAPEG residue of 'D0351' transgenic plant. a: Quantitative analysis of PM residues in 'D0351' $T_{0}$ transgenic plants using gas chromatography; b: Quantitative analysis of PM residues in 'D0351' $T_{1}$ transgenic plants using gas chromatography. The "** presents the value is extremely significant at 0.05 level based on student $t$ test; the "*** presents the value is extremely significant at 0.01 level based on student $t$ test 
wild-type levels, respectively, and these differences were significant. In addition, the residue abundance in these plants at $48 \mathrm{~h}$ was 1.43 -fold higher than that in the wild-type plants, and this difference was extremely significant.

The trend found for the variation in the PM residue abundance in CsMAPEG T1-generation transgenic plants was consistent with that found in the T0-generation plants and was characterized by an initial increase followed by a decrease. The residue abundances in the CsMAPEG(+)overexpressing plants were lower than those in the wildtype plants at the six tested time points. After reaching the maximal value of $0.11 \mathrm{mg} / \mathrm{kg}$ at $24 \mathrm{~h}$, the residue abundance showed a significantly decreasing trend. The values found for the CSMAPEG(+)-overexpressing plants at 6 and $48 \mathrm{~h}$ were 0.66 - and 0.62 -fold of the wild-type values, respectively, and these differences were significant; in addition, an extremely significant difference, which corresponded to a 0.52 -fold change, was found at $72 \mathrm{~h}$. The average PM residue abundance in CsMAPEG(+)-overexpressing T1-generation plants was $0.07 \mathrm{mg} / \mathrm{kg}$, which was lower than that found for the wild-type plants $(0.02 \mathrm{mg} /$ $\mathrm{kg}$ ). The residue abundance in the CsMAPEG(-)-overexpressing plants was higher than that in the wild-type plants at all tested time points, and significant 1.66- and 1.36 -fold changes were detected at 1 and $12 \mathrm{~h}$, respectively. The average residue abundance in the CsMAPEG(-)-overexpressing plants was $0.11 \mathrm{mg} / \mathrm{kg}$, which was $0.02 \mathrm{mg} / \mathrm{kg}$ higher than that found for the wild-type plants.

\section{Physiological and biochemical indexes under PM treatment \\ POD analysis of CSMAPEG-overexpressing plants}

As shown in Fig. 8a and b, most treatments of $\mathrm{T}_{0}$ plants resulted in a trend for enzyme activity consisting of an increase followed by a decrease, and the POD enzyme activity reached a maximal level after 6 days. A comparison of the 'D0351' and 'D9320' wild-type plants treated with distilled water showed that the enzyme activity in 'D0351' plants at each time point was higher than that in 'D9320' plants, which indicated that POD responds to PM in a low-residue-abundance cucumber cultivar. The enzyme activity of the 'D0351' CSMAPEG(-)-overexpressing plants was lower than that of the 'D0351' wildtype plants at the various time points after PM treatment. After PM treatment, the 'D9320' CsMAPEG(+)-overexpressing plants exhibited higher activity than the 'D9320' wild-type plants at all tested time points with the exception of $48 \mathrm{~h}$. These results indicated that the alternation in CsMAPEG expression changed the POD enzyme activity in plants, CsMAPEG overexpression enhanced POD enzyme activity, and antisense CSMAPEG weakened POD enzyme activity.
The change in the POD content in the T1-generation fruits was the same as that found in the T0-generation fruits (Fig. 9a and b). 'D9320' CsMAPEG(+)-overexpressing plants showed a maximum POD value of $50.48 \triangle \mathrm{OD} 470 \bullet \mathrm{min}-1 \cdot \mathrm{g}-1 \mathrm{FW}$ at 6 days. The maximal difference in activity between the 'D9320' CsMAPEG(+)overexpressing and wild-type plants after PM treatment appeared on day 4, and the POD activity of the CsMAPEG(+)-overexpressing plants was 1.19-fold higher than that of the wild-type plants. The POD activity of the 'D0351' CsMAPEG(-)-overexpressing plants was similar to that of the wild-type plants after PM treatment, although the POD content in these fruits was lower than that of the 'D0351' CSMAPEG(+)-overexpressing plants, and this difference was highly significant.

\section{GST analysis of CSMAPEG-overexpressing plants}

As shown in Fig. 8c and d, the GST content of the 'D0351' wild-type plants treated with distilled water first increased, reaching a maximum value of $191.14 \mathrm{U} \cdot \mathrm{mg}-1$ on day 6, and then decreased. However, the GST content of the 'D9320' wild-type plants treated with distilled water decreased continuously. The GST contents in the two cucumber genotypes showed different trends; thus, GST might be related to the detoxification of PM in cucumber fruits. After PM treatment, the GST content of 'D0351' CsMAPEG(-)-overexpressing plants was lower than that of the wild-type plants at all tested time points with the exception of day 6. In addition, after PM treatment, the GST content of the 'D9320' CsMAPEG(+) plants at each time point was significantly higher than that of the 'D9320' wild-type plants, which indicated that the GST content was affected by the expression of CsMAPEG. Plants overexpressing CsMAPEG exhibited a high GST content, whereas plants overexpressing antisense CSMAPEG showed a low GST content.

The GST content in the T1 fruits from 'D0351' CsMAPEG(-)-overexpressing plants was lower than that in 'D0351' wild-type plants at most time points after PM treatment. The GST content of 'D9320' CsMAPEG(+)-overexpressing plants was higher than that of the wild-type plants at most tested time points after PM treatment, and the general trend was similar to that found for the T0-generation plants (Fig. 9c and d).

\section{SOD analysis of CSMAPEG-overexpressing plants}

As shown in Fig. 8e and f, the SOD content of the 'D0351' wild-type plants treated with distilled water increased slightly starting from day 2 and reached a maximum of $264.98 \mathrm{U} \cdot \mathrm{g}-1 \mathrm{FW}$ on day 4 , whereas that of the 'D9320' wild-type treated with distilled water showed a continuous decline. These findings indicated that SOD was directly involved in the metabolic response to the pesticide PM, and the difference in its content might be 
A

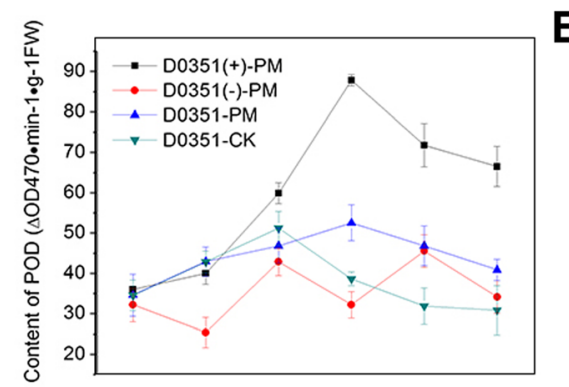

C

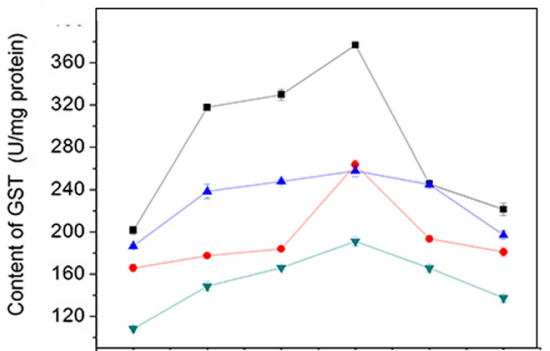

E

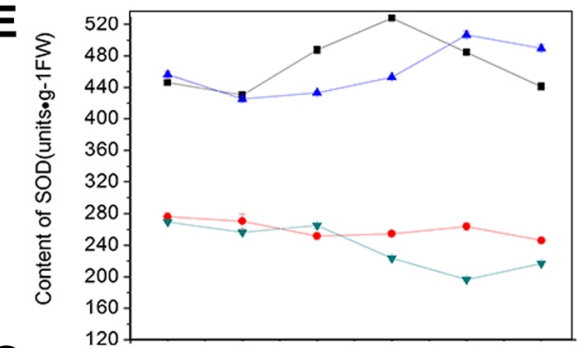

G

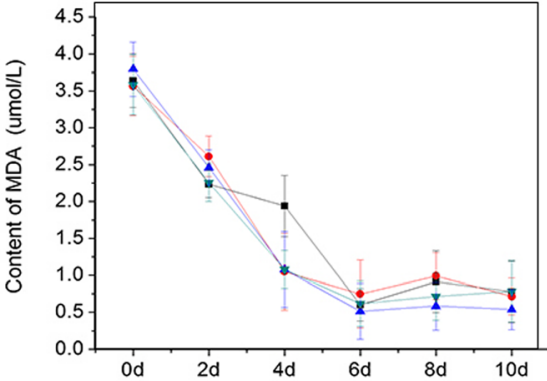

B
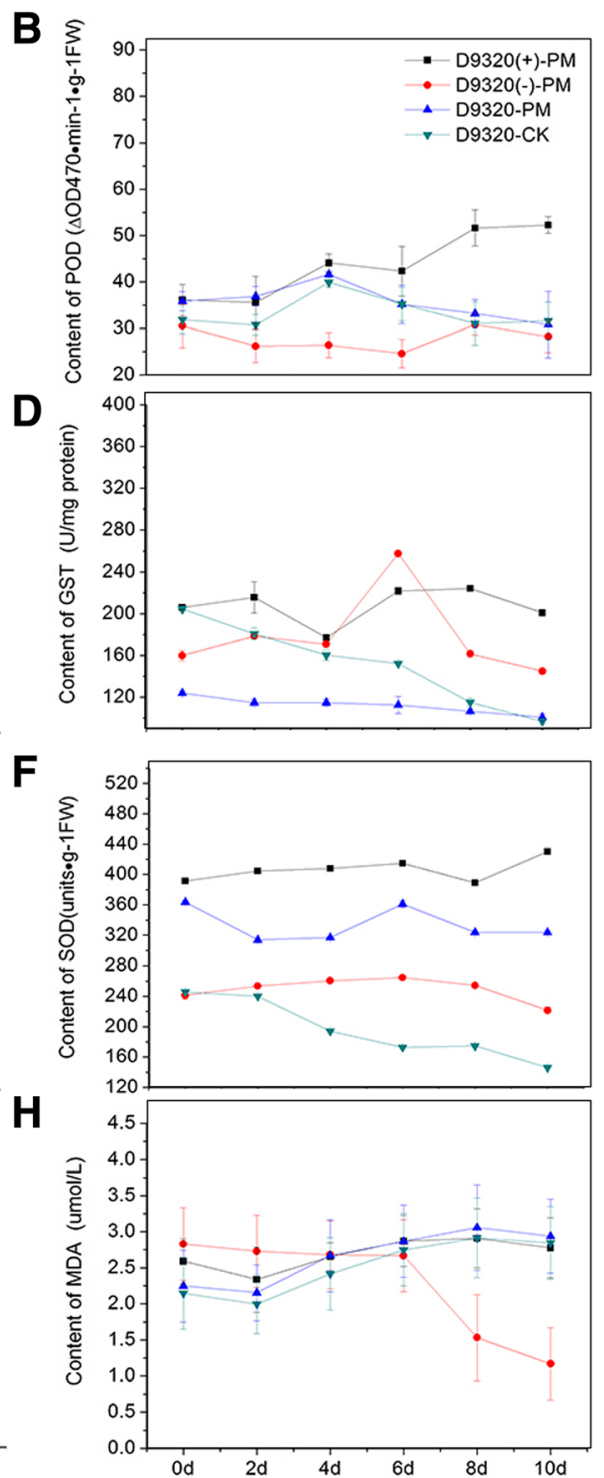

Fig. 8 Analysis of physiological indexes under To stress of PM. a: '0351' POD content; b: '9320' POD content; c: '0351' GST content; d: '9320' GST content; e:'0351' SOD content; f: '9320' SOD content; g: '0351' MDA content; $\mathbf{h}$ : '9320' MDA content

one of the reasons for the difference in PM residue abundance found between fruits of the two varieties. After PM treatment, the SOD contents of the 'D0351' CSMAPEG(-)-overexpressing plants were lower than those of the 'D0351' wild-type plants at all tested time points. The SOD contents of the 'D9320' CsMAPEG(+)overexpressing plants at all tested time points after PM treatment were higher than those of the 'D9320' wildtype plants. These results showed that the expression of CsMAPEG affected the SOD content in cucumbers.

The SOD content in the T1-generation plants was lower compared with that of the T0-generation plants at different time points, although the overall trend remained unchanged. After PM treatment, the SOD content of the
'D0351' CsMAPEG(-)-overexpressing plants was lower than that of the 'D0351' wild-type plants at each tested time point. In addition, the SOD content of the 'D9320' CSMAPEG(+)-overexpressing plants was basically the same as that of the 'D9320' wild-type plants after PM treatment (Fig. 9e and f).

\section{MDA analysis of CSMAPEG-overexpressing plants}

As shown in Fig. 8g and h, the change in the MDA content after PM exposure showed differences between the 'D0351' and 'D9320' varieties. The MDA content in 'D0351' decreased rapidly, reached the lowest value after 6 days, and then slightly increased. In 'D9320' plants, the MDA content decreased slightly during the first 2 days 

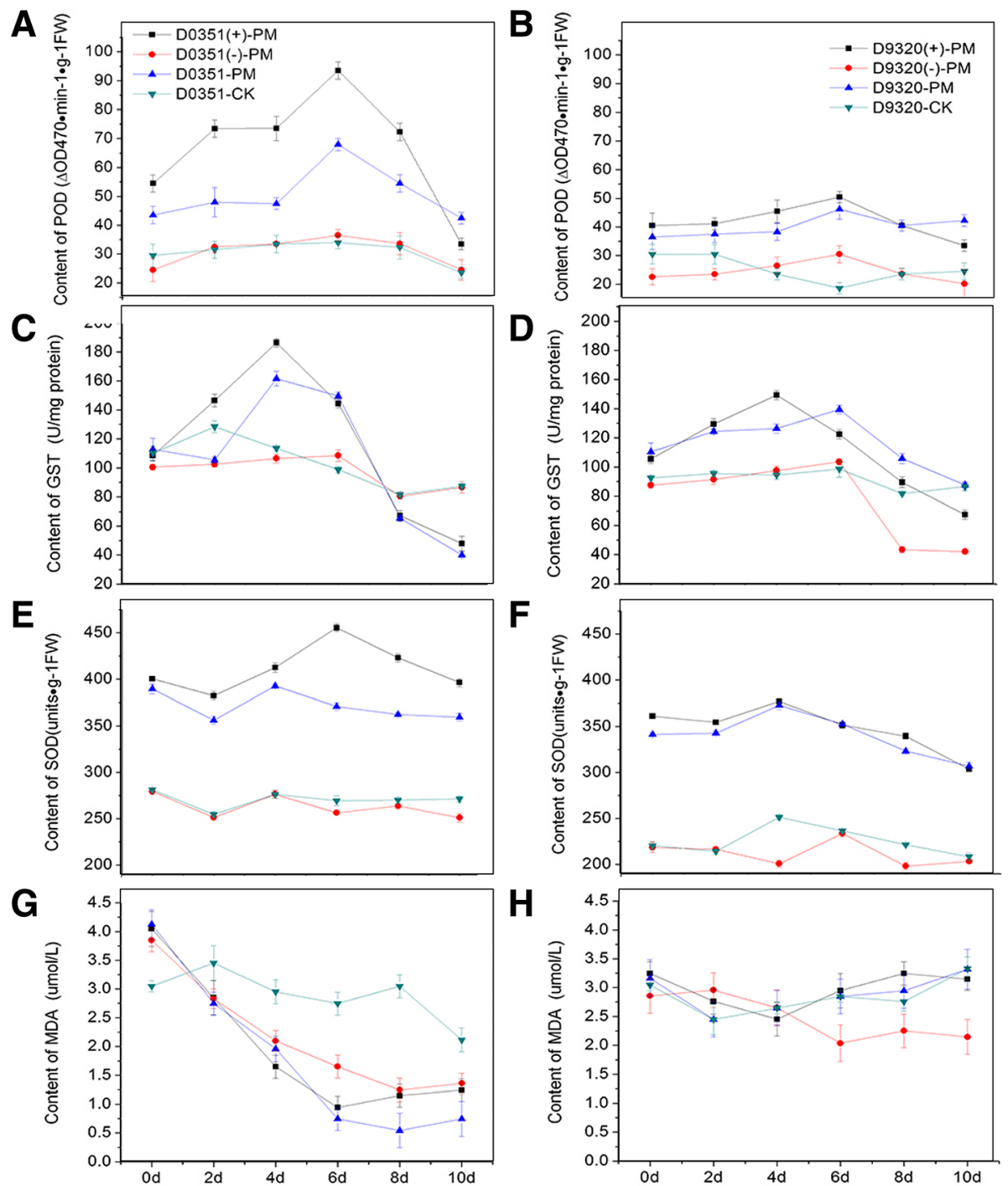

Fig. 9 Analysis of physiological indexes under T1 stress of PM. a: '0351' POD content; b: '9320' POD content; c: '0351' GST content; d: '9320' GST content; e:'0351' SOD content; f: '9320' SOD content; g: '0351' MDA content; $\mathbf{h}$ : '9320' MDA content

and then increased substantially. 'D0351' and 'D9320' plants were subjected to four different treatments, namely, the transfection of sense or antisense constructs and the spray administration of distilled water or PM. The results showed that the MDA contents in the same variety were similar and that the differences in the MDA content following the different treatments were very small. The trend obtained for the T1-generation plants was the same as that found for the T0-generation plants, and the MDA content showed slight differences (Fig. 9g and $\mathrm{h}$ ). These findings indicated that the MDA content in cucumber fruits was only affected by cultivar differences and that CSMAPEG had no effect on the content of MDA. Thus, although MDA is involved in the stress response, its metabolic effect was not significant after PM treatment.

\section{Discussion}

A transcriptome analysis showed that exogenous PM can activate the GST metabolism pathway in cucumber, including the MAPEG family, which is a newly discovered microsome protein superfamily. Most members of this family participate in the metabolism of exogenous lipids and have specific detoxification and antioxidation functions. For example, mGST1, which is a phase II drug-metabolizing enzyme that belongs to the MAPEG family, has the following two functions in vivo: as a glutathione S-transferase, it catalyzes the metabolism of lipophilic and electrophilic substrates and decreases the toxicity and increases the excretion of the latter in vitro, and as a GST-dependent peroxidase, it catalyzes the combination of oxidative stress products and GSH and exerts anti-lipid peroxidation effects. Thus, this protein 
plays an extremely important role in the stability of the internal plant environment [28].

Analyzing the expression patterns of genes is important for the elucidation of gene functions. A significant difference in the expression patterns of CSMAPEG was observed between cucumbers with different genotypes. Following PM exposure, the expression of this gene was significantly upregulated at all time points in 'D0351' and downregulated at most time points in 'D9320'. The expression of CsMAPEG in the roots, stems, leaves and fruits of 'D0351' was significantly higher than that in the same organs of 'D9320' at the same time points. The stems and leaves were sprayed with PM, and the results showed that these organs are involved in PM transport, as reflected by the finding that the most obvious differences in expression were found in these organs. These results are consistent with the findings obtained in a transcriptome sequencing analysis performed in a previous study [12]. Our findings indicated that CsMA$P E G$ was specifically upregulated in the low-residueabundance cucumber cultivar 'D0351' and responded positively to PM exposure. Thus, this gene is an important mechanism for the differences in the residue abundances between the two cucumber genotypes. By analyzing the expression of CsMAPEG in different tissues and organs of 'D0351' and 'D9320' after PM stress, we found that CsMAPEG was constitutively expressed and showed obvious tissue differences, particularly in the leaves, stems and fruits. The CsMAPEG expression levels in different tissues and organs were ordered as follows: fruits $>$ leaves $>$ stems $>$ roots. Previous research analyzed the expression patterns of CsABC19 in 'D0351' and 'D9320' under PM stress, and the results showed that the CsABC19 gene was mainly expressed in 'D0351' [13], and this previous study revealed similar expression patterns for the CsMAPEG and CsABC19 genes. Another study showed that the $C s A B C 19$ gene was mainly expressed in cucumber fruits, and the results of the tissue-specific analysis performed in the experiment also demonstrated that fruits exhibited the highest relative expression. Based on the above-presented results, CSMAPEG can actively respond to PM stress and might promote tolerance to PM and participate in the detoxification of pesticides.

The 2000-bp promoter sequence upstream of the CSMAPEG starting codon was obtained from the cucumber genome database, and the promoter sequence of CSMAPEG was analyzed. Many light-response elements, which are regulated by light signals, were found in the promoter region of this gene. Many hormone-responsive elements, such as the TCA element, the GARE motif, and ERE cis-regulatory elements, can regulate SA, GA, ethylene and other hormones. Hormones can play an important role in the adaptation and signal transduction of the plant stress response. SA can induce an increase in membrane protective enzymes (mainly SOD and POD) in cucumber leaves, and these enzymes scavenge oxygen free radicals and enhance the antioxidant capacity of plants [29]. Under stress conditions, the GA synthesis system can be activated to synthesize high levels of GA and thereby enhance the ability of plants to resist stress [30]. JA is an endogenous growth regulator in higher plants. During stress resistance, the signal molecules inducing the expression of resistance genes are closely related to plant resistance [31]. In this study, we found that the expression patterns of CsMAPEG showed significant differences between 'D0351' and 'D9320' after SA and GA induction. 'D0351' was more sensitive to hormone induction than 'D9320', and this finding might be related to the low PM residue abundance in 'D0351'. However, further experiments are needed to confirm whether the resistance of cucumber to PM stress can be enhanced by regulating the hormone pathway and subsequently the metabolism of PM. The CsMAPEG gene sequence also contains many defense and stress-induced response regulatory elements, which might play an important role in plant resistance to PM. Stress resistance can induce plant resistance, and new varieties with varying levels of stress and disease resistance have been screened under stress conditions. Under drought and low temperature stress conditions, the expression patterns of CsMAPEG in 'D0351' and 'D9320' were the same, and no difference was found among the cultivars. However, the relative expression of CSMAPEG in 'D0351' was significantly higher than that in 'D9320', and 'D0351' was more responsive to drought and low temperature stress than 'D9320'. The expression of CsMAPEG after Cor stress was significantly higher than that obtained after the other two stress treatments, which suggested that this gene is responsive to Corynespora leaf spot and might be involved in disease resistance. It has been confirmed that 'D9320' shows high resistance to Cor, and the expression of CsMAPEG in 'D9320' was higher than that in 'D0351' under Cor stress. Thus, CsMAPEG might play a role in resistance to Corynespora cassiicola, but whether the gene is a disease-resistance gene and the specific regulatory mechanism of cucumber resistance to this disease need to be further verified.

Because fruits constitute the edible part of cucumber plants and exhibit the highest CSMAPEG expression, fruits from the T0- and T1-generation plants with the same growth potential and similar CSMAPEG expression were selected for the detection of PM residues. A comparative analysis of the data shown in Figs. 6 and 7 reveals that the PM residue abundances in the 'D0351' T0-generation, T1-generation and wild-type plants were lower than those in the 'D9320' plants, which indicated 
that 'D0351' is a cucumber variety with a lower PM residue abundance compared with 'D9320'. This result is consistent with those reported by Liu [9] and Wu [2]. Based on the above-described analysis (Fig. 4), CsMA$P E G$ expression in 'D9320' increased rapidly starting at $12 \mathrm{~h}$. After $12 \mathrm{~h}$, the PM residue abundance in CsMA$P E G(+)$-overexpressing plants was significantly or extremely significantly lower than those in the CsMA$P E G(-)$-overexpressing and wild-type plants, which indicated that the PM residue level was negatively correlated with the CSMAPEG gene expression level. Zhao Wen et al. studied the rate of change in the pesticide residue abundance in fruits. The highest rate of change in the pesticide residue abundance in cucumber fruits was detected at $24 \mathrm{~h}$ [11]. In the T0 generation, the most significant differences among CsMAPEG(+)-overexpressing, CsMAPEG(-)-overexpressing and wild-type plants were found at $24 \mathrm{~h}$. After $24 \mathrm{~h}$, the residue abundances of the CsMAPEG(+)-overexpressing plants showed a lower decrease. The T1-generation plants showed the most significant difference at $72 \mathrm{~h}$, and the CsMAPEG(+)-overexpressing plants showed obvious upward and downward trends at each time point. These results might be due to differences in the genetic process of T1-generation plants. CsMAPEG can effectively reduce the PM residue abundance in 'D0351' and 'D9320' T1-generation plants, which indicated that the gene exhibits certain genetic stability. CsMAPEG can respond to PM stress, and CSMAPEG(+) overexpression plays an important role in reducing the PM residue abundance. This gene can effectively reduce the abundance of PM residues and might be related to detoxification and metabolism in cucumber. The transfection of antisense genes increased the PM residue abundance in fruits, which indicated that antisense genes play an inverse regulatory role. The transfection of the antisense gene might have inhibited degradation- and metabolismrelated enzymes or genes and affected the detoxification metabolism of PM in cucumber fruits. This result is consistent with those reported by Liu [14].

In our study, the transfer of CSMAPEG(+) into cucumber increased the SOD and POD contents in cucumber fruits, and the transfer of antisense CSMAPEG(-) into cucumber resulted in significantly lower SOD and POD contents compared with those found in the corresponding wild-type control. These findings indicated that CSMAPEG affected the SOD and POD contents in cucumber. The SOD and POD contents could be regulated by increasing the expression of CSMAPEG and might induce the accumulation of $\mathrm{H}_{2} \mathrm{O}_{2}$ in cucumber fruits. The metabolism of PM and the effective reduction of pesticide residues in cucumber might be improved by the reduction of $\mathrm{H}_{2} \mathrm{O}_{2}$ to $\mathrm{H}_{2} \mathrm{O}$. The results were consistent with those reported by Chaitanya [32], Mazorra [33], and Davies [34] as well as those obtained in other studies on POD activity after abiotic stress. Our results showed that the variation in the MDA content in plants of the same variety was similar and that the difference in the MDA content was very small. The MDA content in cucumber fruits was greatly affected by the variety, although CsMAPEG had little effect on the content of MDA. Moreover, MDA is involved in the stress response, and detoxification is the first important function of GST identified in organisms. Plants can self-detoxify toxic chemicals in the body, and GST plays an important role in this process [22]. In addition, the GST content in cucumber increased significantly under abiotic stresses, and this finding indicated that GST can also act as a cellular signal in plant stress tolerance. The research results showed that the GST trends in the high- and low-residue-abundance cultivars were different and that GST might be involved in the detoxification metabolism of PM in cucumber fruits. CsMAPEG overexpression affected the GST content in cucumber, and the opposite trend was found for antisense CsMAPEG. These findings indicated that the transfer of CsMAPEG(+) into cucumber could increase the GST levels and that GST could detoxify cucumber fruits and reduce the damage caused by PM. These results correspond to the upregulation of GST-related genes and the significant enrichment of the GSH metabolism pathway detected in the analysis of gene expression profiles [12]. PM stress might activate the GSH metabolism pathway in cucumber. The role of CSMAPEG in the pathway could enhance the activity of protective enzymes in the membrane system, enhance the antioxidant capacity of cucumber and stabilize the internal environment of cucumber. This protein can also increase the GST content, and GST catalyzes the metabolism of PM, thereby reducing the toxicity of PM, increasing its discharge in vitro, and reducing the damage caused by PM residues.

\section{Conclusions}

In summary, the subcellular localization of CsMAPEG was found to be cytoplasmic, which identified this protein as a cytoplasmic protein. CSMAPEG displayed differences between varieties and tissues, was strongly expressed in 'D0351' and showed its highest expression in fruits. CSMAPEG can respond to SA, GA and Cor and might play an important regulatory role in the response of plants to abiotic and biological stresses. The overexpression of CsMAPEG(+) in cucumber effectively reduced the PM residue abundance, increased the activities of protective enzymes (POD and SOD) in the cucumber inner membrane system and increased the activity of GST, which has detoxification functions. The transfer of antisense CsMA$P E G(-)$ into cucumber had the opposite effects. In brief, the results showed that CsMAPEG plays an important role 
in reducing the PM residue abundance in cucumber. This study provides a valuable foundation for further study of the molecular mechanisms responsible for the low accumulation of PM residues in cucumber. Ultimately, a greater understanding of these molecular mechanisms will enable targeted breeding strategies for the development of cucumber varieties with low pesticide residues and increased value.

\section{Methods}

\section{Plant materials and stress treatments}

The homozygous cucumber lines 'D0351' (with a low PM residue abundance) and 'D9320' (with a high PM residue abundance) were selected as the experimental materials $[2,9]$. Seeds of these lines were provided by the cucumber research group at Northeast Agricultural University, Harbin, China. 'D0351' and 'D9320' seeds were planted in soil at the Biotron in March 2013 under the following conditions: day/night temperatures of 28/ $18^{\circ} \mathrm{C}$, 12-h day/12-h night cycle, and $70 \%$ relative humidity. Conventional barrel-and-frame cultivation methods were adopted for unified management. Once the cucumber was ripe at the 10th node, the plants were sprayed with 400x PM solution until the surface of the leaves and fruits began to drip, and the control group was sprayed with the same amount of distilled water. Samples were obtained 1, 6, 12, 24, 48 and $72 \mathrm{~h}$ after treatment, frozen in liquid nitrogen and stored at $80^{\circ} \mathrm{C}$ for gene expression analysis.

Three true-leaf seedlings were used to determine the changes in CSMAPEG expression after treatment with SA (foliar spraying with $0.5 \mathrm{mmol} / \mathrm{L} \mathrm{SA}$ [35]), JA (foliar spraying with $100 \mu \mathrm{mol} / \mathrm{L} \mathrm{JA}$ ), GA (foliar spraying with $100 \mu \mathrm{mol} / \mathrm{L}$ GA [14]), drought stress (seedlings were irrigated with $50 \mathrm{~mL}$ of $40 \%$ PEG4000, and leaves were harvested 8 days after treatment), Corynespora cassiicola Wei (Cor) stress (disease stress; seedlings were sprayed with $1 \times 10^{5}$ colony-forming units $/ \mathrm{mL}$ Cor, and leaves were harvested $24 \mathrm{~h}$ after treatment) and cold (lowtemperature) stress (the day/night temperatures were set to $10 \pm 0.5^{\circ} \mathrm{C} / 5 \pm 0.5^{\circ} \mathrm{C}$, the illumination time was $16 \mathrm{~h}$, the light intensity was $4000 \mathrm{~lx}$, and the stress was applied for 10 days). Five plants with identical growth were selected as replicates of each treatment, and leaves were harvested $24 \mathrm{~h}$ after treatment, frozen with liquid nitrogen and stored at $-80{ }^{\circ} \mathrm{C}$ for analysis of the expression patterns induced by external factors.

T0-generation CsMAPEG(+) and CSMAPEG(-) transgenic cucumber plants with similar expression levels were sprayed with $4 \mathrm{mMPM}$ solution. Leaves were harvested 1, 6, 12, 24, 48 and $72 \mathrm{~h}$ after treatment. Wildtype 'D9320' plants were sprayed as controls. Seeds of the T1-generation plants were obtained through selfcrossing of the T0-generation lines. The T1-transgenic plants were treated using the same protocols as those used for the T0-generation plants.

\section{Cloning and bioinformatics analysis of CSMAPEG}

The full-length coding sequence (CDS) of CSMAPEG was obtained by BLAST alignment using the cucumber genome database (http://www.icugi.org/). Primer Premier 5.0 software (PREMIER Biosoft International, CA, USA) was used to design the primers for cloning the CDS of CsMAPEG (geneIDCsa5G409710.1): CsMAPEG-F, ATGGCC GCAATCCAGCTTCTC; and CsMAPEG-R, TTAATG ACGAAGAAGCTTAACACCGAAC. PCR amplification was performed using 'D0351' cDNA as the template and the following temperature program: $94{ }^{\circ} \mathrm{C}$ for $5 \mathrm{~min}, 35$ cycles of $94^{\circ} \mathrm{C}$ for $30 \mathrm{~s}, 58^{\circ} \mathrm{C}$ for $30 \mathrm{~s}$ and $72{ }^{\circ} \mathrm{C}$ for $30 \mathrm{~s}$ and $72{ }^{\circ} \mathrm{C}$ for $10 \mathrm{~min}$. The amplified PCR products were detected by agarose gel electrophoresis, and a colloid recovery kit (TransGen Biotech) was used to recover the target bands. The recovered fragments were attached to the T3 vector (TransGen Biotech) using the following linkage system: $4 \mu \mathrm{L}$ of the target gene and $1 \mu \mathrm{L}$ of the T3 ligase were reacted at room temperature $\left(20^{\circ} \mathrm{C}-37^{\circ} \mathrm{C}\right)$ for 5 min. After the reaction, the sample was transformed into Escherichia coli DH5 $\alpha$ competent cells by thermal shock, and the cells were then incubated overnight on LB agar plates containing X-Gal, IPTG and Amp at $37^{\circ} \mathrm{C}$. Single white bacterial colonies were isolated and sent for sequencing at GENEWIZ.

The CSMAPEG protein sequence was analyzed using the Conserved Domain Database (CDD) of NCBI (https://www.ncbi.nlm.nih.gov/), and ProtParam (https:// web.expasy.org/cgi-bin/protparam/protparam) was used to assess the physicochemical properties of the amino acids. The ProtScale website (https://web.expasy.org/ protscale/) was used to predict the hydrophilicity of the gene-encoded proteins. Online prediction of the protein transmembrane structure was performed using TTMHMM Server v.2.0. The signal peptide prediction of cucumber proteins was performed using SignalP 4.1 Server (http:// www.cbs.dtu.dk/services/SignalP/), and the protein secondary structure was predicted using the SPOMA online tool (https://npsa-prabi.ibcp.fr/cgi-bin/npsa_automat.pl? Page = npsa_sopma.html). The phylogenetic tree was constructed using the neighbor-joining method with MEGA 5.2 software.

\section{Real-time quantitative reverse transcription-polymerase chain reaction (qRT-PCR) analysis}

Total RNA from $100 \mathrm{mg}$ of fresh cucumber leaves was extracted using the TRIzol reagent (Invitrogen ${ }^{\mathrm{m}}$ ) and used for qRT-PCR analysis after ground in a sterilized grinding bowl with liquid nitrogen, and the RNA purity was detected by gel electrophoresis. A reverse transcription kit (Toyobo, Japan) was used for the reverse transcription of 
RNA into single-stranded cDNA, and the products were stored at $-20^{\circ} \mathrm{C}$ for further analysis. The following primers were designed using the online tool at https:// www.genscript.com/tools/real-time-pcr-tagman-primerdesign-tool: CsMAPEG-qF, CGCGCTCGCAAGAAGTATAA; and CsMAPEG-qR, GAAGCGGTAAGGCAAGGA TG. qRT-PCR was performed using SYBR Green Master Mix (Toyobo, Japan), and the reaction system consisted of $10 \mu \mathrm{L}$ of SYBR Green PCR Master Mix, $0.5 \mu \mathrm{L}$ of the upstream primer, $0.5 \mu \mathrm{L}$ of the downstream primer, $2 \mu \mathrm{L}$ of cDNA template, and $\mathrm{ddH}_{2} \mathrm{O}$ to a total volume of $20 \mu \mathrm{L}$. The amplification conditions were as follows: denaturation at $95^{\circ} \mathrm{C}$ for $10 \mathrm{~min}$ followed by 40 cycles of $95^{\circ} \mathrm{C}$ for $15 \mathrm{~s}$ and $55^{\circ} \mathrm{C}$ for $15 \mathrm{~s}$. Relative quantification of gene expression was performed using CsEF1 $\alpha$ [36] (GenBank accession number: XM_004138916) as the control (CsEF1a-qrF: CCAAGGCAAGGTACGATGAAA, CsEF1aqrR: AGAGATGGGAACGAAGGGGAT). Four replicates of each treatment were used. A melting-curve analysis was performed after the amplification was completed. The 2$\Delta \triangle \mathrm{CT}$ method [37] was used to analyze the real-time qPCR results.

\section{Sequence analysis of the CSMAPEG promoter}

The 2000-bp promoter region upstream of CsMAPEG was obtained from the cucumber gene database (http:// www.icugi.org/), and the cis-acting elements in the promoter region were analyzed using the online tool PlantCARE (http://bioinformatics.psb.ugent.be/webtools/ plantcare/html/) [38].

\section{CSMAPEG subcellular localization}

The following primers were designed with XmaI and BamHI restriction sites: CsMAPEG-LF, CGGGATCCAT GGCCGCAATCCAGCTTCTC; and CsMAPEG-LR, CCCCCCGGGTAATGACGAAGAAGCTTAACA

CCGAAC. The CsMAPEG open reading frame without a stop codon was amplified. The pEASY-T3-CsMAPEG fusion expression vector and pGII-eGFP transient expression vector were digested by rapid digestion with the endonucleases XmaI and BamHI, and the purified product was recovered by gel electrophoresis and ligated using the T4 ligase to obtain the fusion expression vector 35S-CsMAPEG-eGFP. This construct was transformed into $E$. coli cells and identified. The empty pGIIEGFP vector was used as a negative control. The 35SCSMAPEG-eGFP and empty pGII-EGFP vector plasmids were transfected into isolated Arabidopsis protoplasts [39]. The subcellular localization in protoplasts was observed using a TCS SP2 confocal spectral microscope imaging system (Leica, Germany). GFP fluorescence was observed at an excitation wavelength of $488 \mathrm{~nm}$ and an emission wavelength of $530 \mathrm{~nm}$.

\section{Transformation of cucumber}

The full-length primers CsMAPEG-F and CsMAPEG-R were used to amplify the target gene by PCR with the pEASY-T3-CsMAPEG plasmid as the template. pCXSN1250 is a plant expression vector that can be used for TA cloning [40]. The PCXSN-1250 vector was digested with $\mathrm{XcmI}$. T4 ligase was used to ligate the target fragment and PCXSN-1250 vector, and the construct was then transformed into $E$. coli. The overexpression vector pCXSN-CsMAPEG(+) and the antisense expression vector pCXSN-CSMAPEG(-) were constructed, and the directionality of the target gene within the vector was confirmed by sequencing. The recombinant plasmid was transferred into Agrobacterium tumefaciens LBA4404 using the freeze-thaw method. The overexpression vector pCXSN-CsMAPEG(+), the antisense expression vector pCXSN-CsMAPEG(-), and empty vectors were simultaneously transferred into 'D0351' and 'D9320' by infestation of the cotyledon nodes of cucumber. Plants transformed with the empty vector were used as controls. After coculture, MS medium containing $1.0 \mathrm{mg} / \mathrm{L}$ glyphosate was used for screening and differentiation. After approximately 20 days, the cotyledonary node began to differentiate, and differentiated buds were observed. Once the differentiated buds matured, they were cut off and transferred to rooting medium. After the main and fibrous roots grew, they were transplanted and domesticated in an incubator [41]. The domesticated transgenic seedlings were planted in a greenhouse, managed uniformly and pollinated to obtain the T1 seeds. The DNA of transgenic cucumber was extracted using the CTAB method. The sequence of the pCXSN vector was used for the primers. The transgenic plants (both the T0 and T1 plants) were identified by PCR and qPCR. The PCR system consisted of $0.5 \mu \mathrm{L}$ of upstream primer $(20 \mu \mathrm{mol} \mathrm{L}-1), 0.5 \mu \mathrm{L}$ of downstream primer $(20 \mu \mathrm{mol} \mathrm{L}-$ 1), $2 \mu \mathrm{L}$ of $10 \times$ PCR buffer (containing $20 \mathrm{mmol} \mathrm{L}-1$ Mg2+), $2 \mu \mathrm{L}$ of dNTPs (10 mmol L-1), $0.2 \mu \mathrm{L}$ of Taq enzyme ( $2 \mathrm{U}), 2 \mu \mathrm{L}$ of cDNA template, and $\mathrm{ddH} 2 \mathrm{O}$ to a total volume of $20 \mu \mathrm{L}$.

\section{Determination of remnant PM}

A mixture of the cucumber leaf sample $(12.5 \mathrm{~g})$ with 25 $\mathrm{mL}$ of acetonitrile was homogenized with a high-speed homogenizer (Heidolph Silent Crusher- $\mathrm{M}^{\circ}$ ) for $2 \mathrm{~min}$ at $15,000 \times g$ and incubated at room temperature for $0.5 \mathrm{~h}$. The homogeneous acetonitrile was extracted into a centrifuge tube containing $3 \mathrm{~g}$ of $\mathrm{NaCl}$, rotated on a vortex for $1 \mathrm{~min}$ and centrifuged for $5 \mathrm{~min}$ at $5000 \times \mathrm{g}$. Five milliliters of the supernatant was dried with a sample concentrator (Termovap) at $60^{\circ} \mathrm{C}$, and $2.5 \mathrm{~mL}$ of acetone was added. The mixture was then filtered through a $0.22-\mu \mathrm{m}$ polypropylene filter. After the filtration membrane solution was clarified, the filter 
membrane solution was allowed to stand for several minutes to observe the presence of turbidity; if particles were present, the solution was passed through the membrane again [42] until no particles remained. An Agilent 7890B5977A gas chromatography system (Agilent Technologies) equipped with a capillary column (HP-5MS, $30 \mathrm{~m} \times 0.25$ $\mathrm{mm} \times 0.25 \mu \mathrm{m}$ ) was used to analyze the level of PM residues. The parameters were as follows: temperature program, maintained at $70^{\circ} \mathrm{C}$ for $5 \mathrm{~min}$, increased to $150^{\circ} \mathrm{C}$ at a rate of $25^{\circ} \mathrm{C} / \mathrm{min}$, increased to $200^{\circ} \mathrm{C}$ at a rate of $3{ }^{\circ} \mathrm{C} /$ min, increased to $280^{\circ} \mathrm{C}$ at a rate of $20^{\circ} \mathrm{C} / \mathrm{min}$ and maintained at $280^{\circ} \mathrm{C}$ for $10 \mathrm{~min}$; sample inlet, $250^{\circ} \mathrm{C}$; non-split injection; flow rate, $1.0 \mathrm{~mL} / \mathrm{min}$; transmission line, $250^{\circ} \mathrm{C}$; four-stage rod, $150^{\circ} \mathrm{C}$; and ion source, $230^{\circ} \mathrm{C}$ [2].

\section{Detection of physiological and biochemical indexes}

A mixture of $0.5 \mathrm{~g}$ of fresh cucumber leaves and $3 \mathrm{~mL}$ of phosphate buffer ( $\mathrm{pH} 7.8$ ) was added to an ice bath and centrifuged for $20 \mathrm{~min}$ at $10,500 \mathrm{rpm}$. This reaction liquid was stored at $4{ }^{\circ} \mathrm{C}$ for the determination of physiological and biochemical indexes.

POD activity was determined using the guaiacol method [43] with minor modifications. Briefly, $112 \mu \mathrm{L}$ of guaiacol was added to $200 \mathrm{~mL}$ of phosphate buffer (pH 6.0), and the mixture was heated until the sufficient dissolution was detected. Subsequently, $19 \mu \mathrm{L}$ of $\mathrm{H}_{2} \mathrm{O}_{2}$ (30\%) was added, and the resulting reaction mixture and stored at $4{ }^{\circ} \mathrm{C}$. Twenty microliters of the reaction mixture was combined with $3 \mathrm{~mL}$ of the reaction liquid to obtain the sample mixture, and $20 \mu \mathrm{L}$ of phosphate buffer mixed with $3 \mathrm{~mL}$ of the reaction liquid was used as the control. The samples were placed in an ultraviolet-visible spectrophotometer (Shimadzu, Japan), and the OD value at $470 \mathrm{~nm} / \mathrm{min}$ (OD values after $0,1,2$, and $3 \mathrm{~min}$ ) was recorded.

SOD activity was analyzed using the NBT method [44] with minor modifications. Reaction mixtures of $\mathrm{H}_{2} \mathrm{O}$, phosphate buffer, Met, NBT, EDTA- $\mathrm{Na}_{2}$ and lactochrome were prepared at a proportion of 5:30:6:6:6:6. Subsequently, $50 \mu \mathrm{L}$ of the reaction mixture was mixed with $3 \mathrm{~mL}$ of the reaction liquid. In addition, $50 \mu \mathrm{L}$ of phosphate buffer mixed with $3 \mathrm{~mL}$ of the reaction liquid was used as the control group, and this control group was divided into two subgroups: control group 1 was treated in the dark, and control group 2 was treated under normal light conditions. The absorbance at OD560 was determined using an ultraviolet-visible spectrophotometer (Shimadzu, Japan).

MDA was detected as described in a previous study [2]. Briefly, $2 \mathrm{~mL}$ of TBA $(0.67 \%)$ was added to $1 \mathrm{~mL}$ of the reaction liquid, and $2 \mathrm{~mL}$ of TBA $(0.67 \%)$ mixed with $1 \mathrm{~mL}$ of distilled water was used as the control group. The orifice of the test tube was sealed to avoid liquid volatilization. After $15 \mathrm{~min}$ of incubation in a boiling water bath, the reaction was cooled and introduced into a centrifugal tube. The samples were centrifuged for $20 \mathrm{~min}$ at $4000 \mathrm{rpm}$ and then detected at 600,532 , and $450 \mathrm{~nm}$ using an ultraviolet-visible spectrophotometer (Shimadzu, Japan).

GST activity was measured based on the method described by Wang Jitao [45] using a kit purchased from the Nanjing Institute of Bioengineering.

\section{Statistical analysis}

All data measurements were replicated at least three times. The data were subjected to statistical analyses using the data processing system Origin 8.0 and DPS 9.5. The data are expressed as the means \pm SDs. The significance of the differences between the treatment and control groups was confirmed by Student's t-tests. The data were analyzed by analysis of variance $(p<0.05$ and $p<0.001$ indicate significant and extremely significant differences, respectively).

\section{Additional files} Additional file 1: Table S1. Locations and sequences of cis-elements in
the promoter regions of the CSMAPEG genes. (DOC $50 \mathrm{~kb}$ )

Additional file 2: Table S2. All sequences data in Additional file 10: Figure S8. (DOC $258 \mathrm{~kb}$ )

Additional file 3: Figure S1. The conserved domain of CSMAPEG coding protein. (PNG $2 \mathrm{~kb}$ )

Additional file 4: Figure S2. Protein hydrophobicity prediction of CSMAPEG. (GIF $10 \mathrm{~kb}$ )

Additional file 5: Figure S3. Signal peptide of CSMAPEG coding protein. (PNG 9 kb)

Additional file 6: Figure S4. The transmembrane region of CSMAPEG coding protein. (PNG $5 \mathrm{~kb}$ )

Additional file 7: Figure S5. Secondary protein structure of CSMAPEG. (PNG $1 \mathrm{~kb}$ )

Additional file 8: Figure S6. Tertiary structure of protein. (PNG 9 kb)

Additional file 9: Figure S7. Phylogenetic tree of CSMAPEG. The amino sequences were subjected to phylogenetic analysis using the neighborjoining method in MEGA5.0 software, with 1000 bootstrap replicates. (JPG $35 \mathrm{~kb}$ )

Additional file 10: Figure S8. pCXSN-CSMAPEG genetic transformation of cucumber. A:cucumber seed; B:CO-culture; C:screening culture; D:plant regeneration; E:rooting culture of resistant seedlings; F:regeneration of resistant seedlings; G:seed of transgenic plants. (PNG $720 \mathrm{~kb}$ )

\section{Abbreviations \\ cDNA: complementrary DNA; CDS: Coding sequences; Cor: Corynespora cassiicola Wei; GA: Gibberellin acid; GFP: Green fluorescent protein; JA: Jasmonic acid; PCR: Polymerase chain reaction; PEG4000: Polyethylene glycol 4000; PM: Propyl-[3-(dimethylamino) propyl]carbamate; qRT-PCR: Real-time quantitative reverse transcription-polymerase chain reaction; RNA: Ribonuncleic acid; RT-PCR: Reverse transcription-polymerase chain reaction; SA: Salicylic acid; Tag-Seq: High-throughput tag-sequencing}

\section{Acknowledgements}

We thank Professor Huazhong Ren (College of Agronomy and Biotechnology, China Agricultural University, Beijing) for providing the method for genetic transformation of cucumber. We acknowledge associate Professor Yongguang Li (Key Laboratory of Northeastern Soybean Biology and Genetic Breeding of the Ministry of Agriculture, China) for providing the pCXSN vector. 


\section{Authors' contributions}

$F Z, Z Q, X Z$ and $M X$ designed experiments. FZ performed the following experiments: expression pattern analysis, transformation of cucumber, determination of PM residues, physiological and biochemical indexes. SL and $\mathrm{J}$ prepared the plant materials, performed RNA extractionand and the subcellular localization. FZ wrote the manuscript and analyzed the data. ZQ revised the manuscript critically for important intellectual content. SL and JL did the analysis and interpretation of data and revised the manuscript. All authors have read and approved the final manuscript.

\section{Funding}

The design of study,collection of samples, analysis and interpretation of data were financially supported by Supporting Certificate of Heilongjiang Postdoctoral Scientific Research Developmental Fund (LBH-Q16021).

\section{Availability of data and materials}

The datasets used and/or analysed during the current study available from the corresponding author on reasonable request. Materials are available by contacting the corresponding author.

\section{Ethics approval and consent to participate}

Not applicable.

\section{Consent for publication}

Not applicable.

\section{Competing interests}

The authors declare that they have no competing interests.

\section{Received: 9 November 2018 Accepted: 13 August 2019} Published online: 22 August 2019

\section{References}

1. Zhang X, Zhang Z, Fan C, et al. Causes and control measures of cucumber downy mildew in solar greenhouse. Modern Agric Sci Technol. 2013;04:149.

2. Wu P. Studies on physiological and molecular basis of low propamocarb residue in cucumber. Harbin: Northeast Agricultural University; 2013.

3. Li C. Cucumber cultivation and main disease control techniques in greenhouse. Chin Veg. 2015;2(35):48-52.

4. Cancer assessment review committee health effects division office of pesticide programs. Evaluation of the carcinogenic potential of ziram (Final report). Washington: Cancer assessment review committee health effects division office of pesticide programs; 2000.

5. Cancer assessment review committee health effects division office of pesticide programs. Third evaluation of the carcinogenic potential of permethrin (final report). Washington: Cancer assessment review committee health effects division office of pesticide programs; 2002.

6. Cancer assessment review committee health effects division office of pesticide programs. Third evaluation of the carcinogenic potential of clodinafop-propargyl (Final report). Washington: Cancer assessment review committee health effects division office of pesticide programs, 1999.

7. Cancer assessment review committee health effects division office of pesticide programs. Evaluation of the mode of action of acetochlor for nasal olfactory epithelium tumors in rats and its relevance to human cancer risk assessment (final report). Washington: Cancer assessment review committee health effects division office of pesticide programs; 2004

8. Liu Y, Zhang J, Cao M, et al. Study and application of enzyme inhibition method for rapid detection of pesticide residues in agricultural products. Mod Pestic. 2004;3(2):25-7.

9. Liu F. Screening of cucumber germplasm resources with low pesticide residues. J Northeast Agric Univ. 2010;07:32-6.

10. Ma B. Determination of remnant residues in cucumber fruits by gas chromatography. J Changjiang Veg. 2010;20:51-3.

11. Zhao W. Comparative analysis of anatomical characteristics of pericarp of low pesticide re sidues in cucumber. China Veg. 2013;20:32-8.

12. Wu P, Qin Z, Zhao W, et al. Transcriptome analysis reveals differentially expressed genesa ssociated with propamocarb response in cucumber (Cucumis sativus L.). Fruit Acta Ph ysiologiae Plantarum. 2013;35(8):2393-406.

13. Meng J, Qin Z, Zhou X, et al. An Atp-binding cassette transporter gene from cucumis sativus L., Csabc19, is involved in propamocarb stress in arabidopsis thaliana. Plant Mol Biol Report. 2016;34(5):947-60.
14. Li S, Qin Z, Xin M, et al. Expression and functional analysis of Cswrky30 in cucumber under propamocarb stress. Sci Agric Sin. 2016;07:1277-88

15. Guo L. Cloning and expression of SDH gene related to residues of Cucumis viride in cucumber. J Northeast Agric Univ. 2016;04:24-33.

16. Liu C, Qin Z, Zhou X. Expression and functional analysis of the propamocarbrelated gene CsDIR16 in cucumbers. BMC Plant Biol. 2018;18:16-28.

17. Cole D. Glutathione transferases in crops and major weeds. In Hatzios K,Kluwer Academic Publishers,Amsterdam, Regulation of Enzymatic Systems Detoxifying Xenobiotics in Plants.1997,385:139-154.

18. Cole DJ, Edwards R. Secondary metabolism of agrochemicals in plants. London: John Wiley and Sons,in agrochemicals and plant protection (roberts T R,ed.); 2000. p. 107-54.

19. Romano M L,Stephensom G R,Tal A. The effect of monooxygenase and glutathione S-transferase inhivitors on the metabolism of diclofopmethyl amd fempxa [rop-ethyl inbarley and wheat. Pestic Biochem Phys1993,46:181-189.

20. Farkas MH, Berry JO. Chlortetracycline detoxification in maize via induction of Glutathione S-transferases after antibiotic exposure. Environ Sci Technol. 2007:1450-6.

21. Hou J, Zhang Q, Zhou Y. Glutaredoxin GRXS16 mediates brassinosteroidinduced apoplastic $\mathrm{H}_{2} \mathrm{O}_{2}$ production to promote pesticide metabolism in tomato. Environ Pollut. 2018;240:227-34.

22. Del RD, Stewart AJ, Pellegrini N. A review of recent studies on malondialdehyde as toxic molecule and biological marker of oxidative stress. Nutr Metab Cardiovasc Dis. 2005;15:316-28.

23. Koca $H$, Bor M, Özdemir F, et al. The effect of salt stress on lipid peroxidation, antioxidative enzymes and proline content of sesame cultivars. environmental experimental botany. 2007, 60(3): 344-351.

24. Xu S, Li J, Zhang X, et al. Effects of heat acclimation pretreatment on changes of membrane lipid peroxidation, antioxidant metabolites, and ultrastructure of chloroplasts in two cool-season turfgrass species under heat stress. Environ Exp Bot. 2006;56(3):274-85.

25. Gémes K, Poór P, Horváth E, et al. Cross-talk between salicylic acid and $\mathrm{NaCl}$-generated reactive oxygen species and nitric oxide in tomato during acclimation to high salinity. Physiol Plant. 2011;142(2):179-92.

26. Zhu D, Du Y, Huang X. MAPEG expression in murine embryonic stem cellderived hepatic tissue system. Stem Cells Dev. 2008:17(4):775-84.

27. Yang $H$, Zheng $X$, Du Y. Kinetics of aristolochic acid I after oral administration of radix aristolochiae or guanxinsuhe preparation in canines. J Ethnopharmacol. 2011;135:569-74.

28. Daniel MM, Said E, Pa RN. Catalysis within the lipid bilayer-structure and mechanism of the MAPEG family of integral membrane proteins. Curr Opin Struct Biol. 2008;18:1-8.

29. Promyoua S, Ketsa S, Wouter G, et al. Salicylic acid alleviates chilling injury in anthurium (Anthurium andraeanum L.)flowers. Postharvest Biol Technol. 2012;64:104-10.

30. Finkelstein RR, Gampala SS, Rock CD. Abscisic acid signaling in seeds and seedlings. Plant Cell. 2002;14:515-45.

31. Hao J, Yi Y, Shang Q, et al. Effect of exogenous salicylic acid on nitrogen assimilation of cucumber seedling under drought stress. Acta Horticulturae Sinica. 2012;01:81-90.

32. Chaitanya KV, Sundar D, Masilamani S, et al. Variation in heat stress-induced antioxidant enzyme activities among three mulberry cultivars. Plant Growth Regul. 2002:36:175-80.

33. Mazorra LM, Nunez $M$, Hechavarria $M$, et al. Influence of brassinosteroids on antioxidant enzymes activity in tomato under different temperatures. Plant Biol. 2002;45:593-6.

34. Davies DG, Swanson HR. Activity of stress-related enzymes in the perennial weed leafy spurge. Environ Exp Bot. 2001;46:95-108.

35. Wu R, Wang L, Wang Z, et al. Cloning and expression analysis of a dirigent protein gene from the resurrection plant boea hygrometrica. Prog Nat Sci Mat Int. 2009;19:347-52.

36. Wan H, Zhao Z, Qian C, et al. Selection of appropriate reference genes for gene expression studies by quantitative real-time polymerase chain reaction in cucumber. Anal Biochem. 2010;399(2):257-61.

37. Schmittgen TD, Livak KJ. Analyzing real-time PCR data by the comparative C(T) method. Nat Protoc. 2008:3:1101-8.

38. Lescot M, Déhais $P$, Thijs $G$, et al. PlantCARE, a database of plant cis-acting regulatory elements and a portal to tools for in silico analysis of promoter sequences. Nucleic Acids Res. 2002;30:325-7. 
39. Yoo SD, Cho YH, Sheen J. Arabidopsis mesophyll protoplasts: a versatile cell system for transient gene expression analysis. Nat Protoc. 2007;2:1565-72.

40. Chen S, Songkumarn P, Liu J, et al. A versatile zero background Tvector system for gene cloning and functional genomics. Plant Physiol. 2009;150:1111-21.

41. Zhang Y, Zhang XL, Liu B, et al. A GAMYB homologue CsGAMYB1 regulates sex expression of cucumber via anethylene- independent pathway. J Exp Bot. 2014;65(12):3201-13.

42. NY/T761-2008, People's Republic of China agricultural industry standard. 2008.

43. Bradford MM. A rapid and sensitive method for the quantitation of microgram quantities of protein using the principle of protein-dye binding. Anal Biochem. 1976;72:248-54.

44. Beauchamp C, Fridovich I. Superoxide dismutase: improved assays and an assay applicable to acrylamide gels. Anal Biochem. 1971:44(1):276-87.

45. Zhou Y, Xia X, Yu G. Brassinosteroids play a critical role in the regulation of pesticide metabolism in crop plants. Sci Rep. 2015;5:9018-25.

\section{Publisher's Note}

Springer Nature remains neutral with regard to jurisdictional claims in published maps and institutional affiliations.

Ready to submit your research? Choose BMC and benefit from:

- fast, convenient online submission

- thorough peer review by experienced researchers in your field

- rapid publication on acceptance

- support for research data, including large and complex data types

- gold Open Access which fosters wider collaboration and increased citations

- maximum visibility for your research: over $100 \mathrm{M}$ website views per year

At BMC, research is always in progress.

Learn more biomedcentral.com/submissions 